\title{
The Usability of Transparent Overview Layers
}

\author{
Donald A. Cox*, Jasdeep S. Chugh *, Carl Gutwin* and Saul Greenberg* \\ Department of Computer Science* and Cognitive Ergonomics Research Laboratory \\ University of Calgary, Calgary, Alberta Canada T2N 1N4 \\ Tel: +1-403-220-6087, E-mail: saul@cpsc.ucalgary.ca
}

\begin{abstract}
Viewports into large visual workspaces are sometimes supplemented by a separate window that displays a miniaturized overview of the entire workspace. Instead of this separate window, we have layered a transparent version of the overview atop the viewport. Because the overview fills the display, it becomes the largest size possible. An exploratory study indicates that people can use this unusual system, where they switch between layers when performing a construction task.
\end{abstract}

Keywords: Transparent interfaces, overviews, groupware.

\section{INTRODUCTION}

Many computer applications provide users with a viewport into a large visual workspace through which they view and manipulate full-sized workspace artifacts. Miniaturized overviews (aka radar views) of the entire workspace are sometimes provided as well [2]. These overviews provide users with global context, which includes the spatial relations between objects, how changes affect objects outside the viewport, and a view rectangle that situates their viewport in the workspace. Overviews can also be active, allowing people to select and manipulate the miniature objects or to drag their view rectangle to a new location. As well, overviews can support awareness in groupware, where participants can see who is in the space, where others are located, and what others are doing $[2,1]$.

Overviews are usually displayed in a separate window. This introduces a usability tradeoff. Because of limited screen space, a large overview window implies a small viewport window, making detailed work more difficult. Yet a small overview window means image fidelity is lost. Also, the physical separation of overview from the viewport sometimes causes people to neglect the overview. To overcome these problems, we are experimenting with transparent layers [3], where the miniature overview is recreated as a layer that is stretched atop the normal viewport [1]. Because the overview fills the display, it becomes the largest size possible. However, visual interference is now present between the two layers.

Cox, D., Chugh, J.S., Gutwin, C. and Greenberg, S. (1998) The Usability of Transparent Overview Layers. CHI'98 Summary Proceedings of the Conference on Human Factors in Computing Systems, p301-302, Late-breaking short paper. ACM Press.
Although others have studied transparency [3], they did not consider the usability of a system that layers an overview atop a viewport. Given that this is a strange way of working, we ran an exploratory study to see whether people could comprehend and successfully use such a system.

\section{THE TESTBED SYSTEM}

Our pipeline construction system lets users select, position and weld 6 types of pipe pieces: T-connectors, right angles, end stubs and 3 different lengths of straight pipes. Welded assemblies act as a single unit. The display contains one of six overview representations layered atop a scrollable viewport (Figure 1). All overviews show all pipe pieces and where they are located, and the overall appearance of the pipeline as it is being constructed. Both overview and viewport are active: any pipeline piece can be selected and dragged to a new location. However, welding is allowed only on the full-sized pieces visible in the viewport layer, which simulates conditions where certain work activities need to be performed in a high fidelity view.

Each overview style represented pipe pieces differently (Figure 2). Five showed the miniature pieces at different levels of (dithered) transparency-0\% (fully opaque), $30 \%$, $50 \%, 70 \%$, and $100 \%$ (fully transparent fill, outline only). A sixth schematic overview showed each piece as a stick figure. The overview in Figure 1 is $70 \%$ transparent.

\section{METHOD}

Eight people participated in this usability study. We observed each user completing a pipeline construction task (including practice) for each of the six overview types. Using a blueprint as a guide, a user assembled a pipeline. They selected from pieces scattered around the workspace, moved them into position, and then welded them. Each task used different pipe layouts, although all comprised the same number and types of pipe pieces. Afterwards, users rankordered their preferences of overview types.

\section{RESULTS AND DISCUSSION}

Our most important finding is that people were able to comprehend and successfully use this unusual system to their advantage. Five specific results are described below.

First, people used the overview repeatedly. They tended to select and reposition pipeline pieces in the overview layer, even when a desired piece type was also visible on the viewport layer. We believe this happened because all piece 


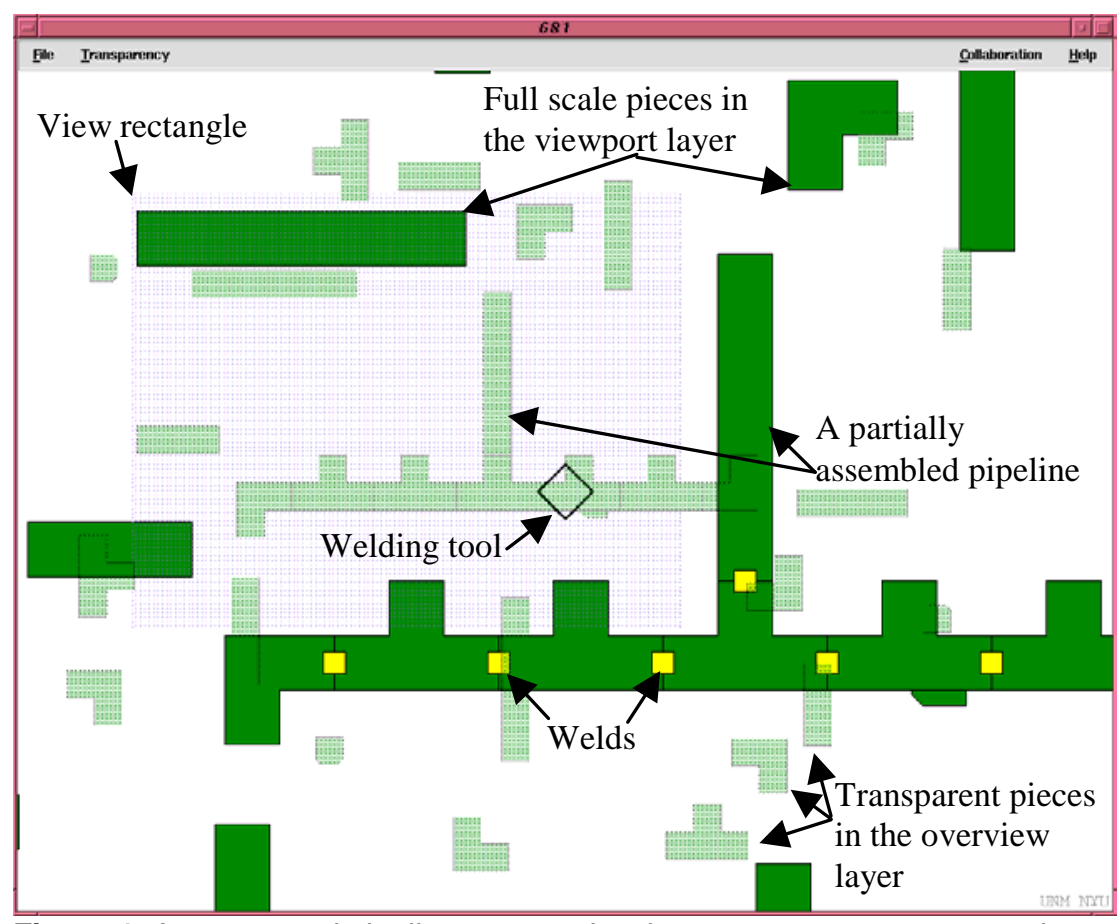

Figure 1: An annotated pipeline system showing a 70\% transparent overview

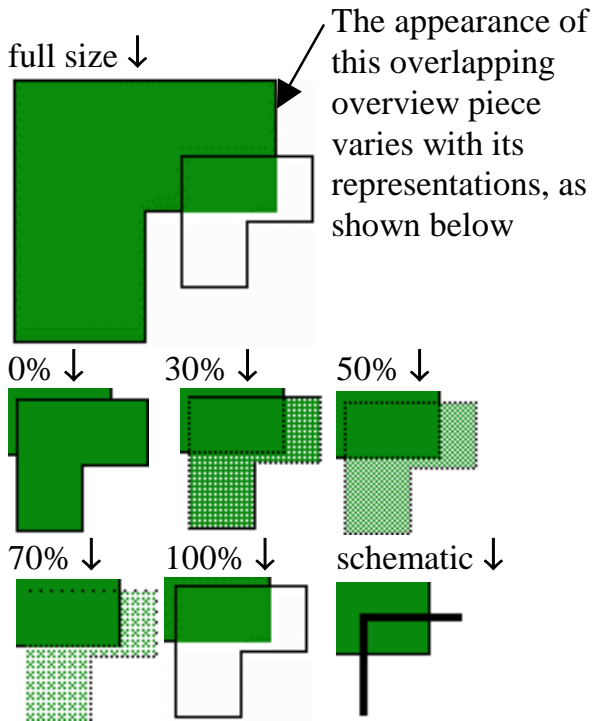

Figure 2: Representations

immediately. While incompatible joins occurred in all overview types, they occurred least with the $70 \%$ and schematic condition, most with $0 \%$ transparency, with the rest falling between the two ranges. Thus when pieces in the overview are visually similar to those in the viewport (i.e. both low and fully transparent pieces), people tended to forget that they were working in two different layers. With visually dissimilar layers (i.e. $70 \%$ transparency and schematic pieces), people were reminded by the pieces' appearance that they were located on different layers.

Our fifth finding concerned people's preferences. People rank-ordered the fully transparent view first, followed by $70 \%$ and $50 \%$ transparency, with the rest receiving low ratings. We believe the schematic rated poorly because people found the thin pieces difficult to select.

In summary, transparent overviews overlaid atop full-scale viewports proved useful, in spite of it being an unusual way of working. Similar to previous studies [3], our combined findings indicate that people worked best with overviews that were $50-75 \%$ transparent. People were also able to shift their focus rapidly between the two views, to the point of initiating an action in the foreground overview layer, and continuing it in the background viewport layer. Of course, further research is required to validate and extend these initial findings.

1 Greenberg, S., Gutwin, C. \& Cockburn, A. Using distortion-oriented displays to support workspace awareness. People \& Computers XI (Proc HCI'96), p281-298, Springer-Verlag, 1996.

2 Gutwin, C. and Greenberg, S. Effects of awareness support on groupware usability. Proc ACM CHI Conf. Human Factors in Computing Systems, 1998.

3 Harrison, B. Design and evaluation of transparent user interfaces. PhD thesis, U Toronto, Ontario, 1996. 